\title{
A Dual Frequency Resonator
}

\author{
P. Lanz, M. Lipnicky, M. Zach \\ TRIUMF, 4004 Wesbrook Mall, Vancouver, B.C. V6T 2 A3
}

\section{Abstract}

A dual frequency resonator system operating at $74 / 37$ $\mathrm{MHz}$ and using nested coaxial conductors for the dee stems in a proton/deuteron isotope production cyclotron is described. The design alternatives are discussed, basic compulations presented, and compared with results from model measurements.

\section{IN'TRODUCTION}

Cyclotrons for production of isotopes used in medical and pharmacentical applications typically deliver proton beams at low to medium energies. In recent years a need for deuteron beams at corresponding energies and lower beam intensities was expressed. The $2 / 1$ mass ratio allows acceleration of both particles in one dee system by selecting the appropriate harmonic number. All available options require changes either to the magnetic field or to the rf frequency when switching between modes.

For reasons of economy, small size, and ease of operation, isotope producing cyclotrons are built as fully automated compact units. A cyclotron with these features (TR30/15), built by Ebco Technologies Ltd. of Vancouver with the design and technical assistance from TRIUMF, is at this time undergoing final acceptance tests at INER* in Taiwan. The dees of this cyclotron are operated at 74 $\mathrm{MHz} / 50 \mathrm{kV}$ for $\mathrm{H}^{-}$, and at $37 \mathrm{MHz} / 25 \mathrm{kV}$ for $\mathrm{D}^{-}$accoleration. A description of the design options and considerations leading to the chosen concept is in (Ref. [1]). This paper deals with one of the options, namely the 'folded line configuration', in more detail.

\section{FOLDED LINE CONCEPTS}

In most cyclotrons the rf resonator system must be located within a very limited space not to adversely affect the stringent requirements on the magnetic field. 'This limitation is even more severe with compact cyclotrons where the overall dimensions are minimized as possible. Resollators operating at low frequencies become long, and the mechanism for switching between frequencies further increases the length. For low operating costs the power dissipated in the rf system must be minimized, and since the skin losses per unit area are highest in the stens and shorting planes, the characteristic impedance of these line sections should be maximized. This requirement is usually in conflict with the permitted tolerances on the magnetic field.

The stcms of the resonator can he rither perpendicular to the cyclotron beam plane or attached to the outer

\footnotetext{
*Institute of Nuclear Fnergy Research, Taiwan, Republic of China.
}

diameter of the dees and extend radially. A layout using the former arrangement is shown schematically in Fig. 1. where the position of the shorting plungers for switching between the high/low frequency modes is indicated in the left/right halves of the symmetrical system.

The operation at the high frequency is shown in Fig. 2. together with the the rf field distribution obtained by SUPERFISH. The moveable shorting plane is also used for frequency adjustments in a broad range of frequencies.

At the low frequency the overall length of the stems that resonate with the capacity of the same dees is substantially shortened by folding the outer conductor by $180^{\circ}$, and using both surfaces of the intermediate cylinder as rf field boundaries. In Fig. 2. is the schematic arrangement and the field distribution.

A second possible solution is shown in Fig. 3. where the operation at the higher frequency is confined to the inner volume between the cylinders. The switching and tuning arrangement is analogous to the one in Fig. 2.

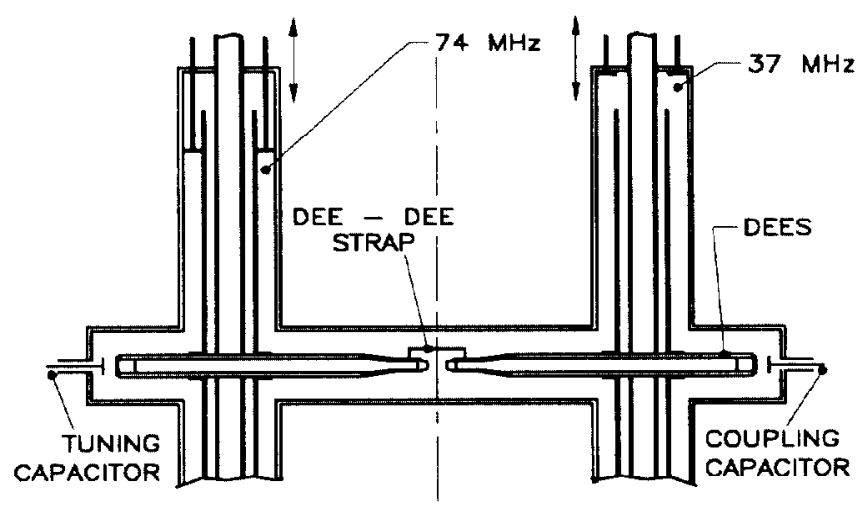

Fig. 1. Resonator layout.

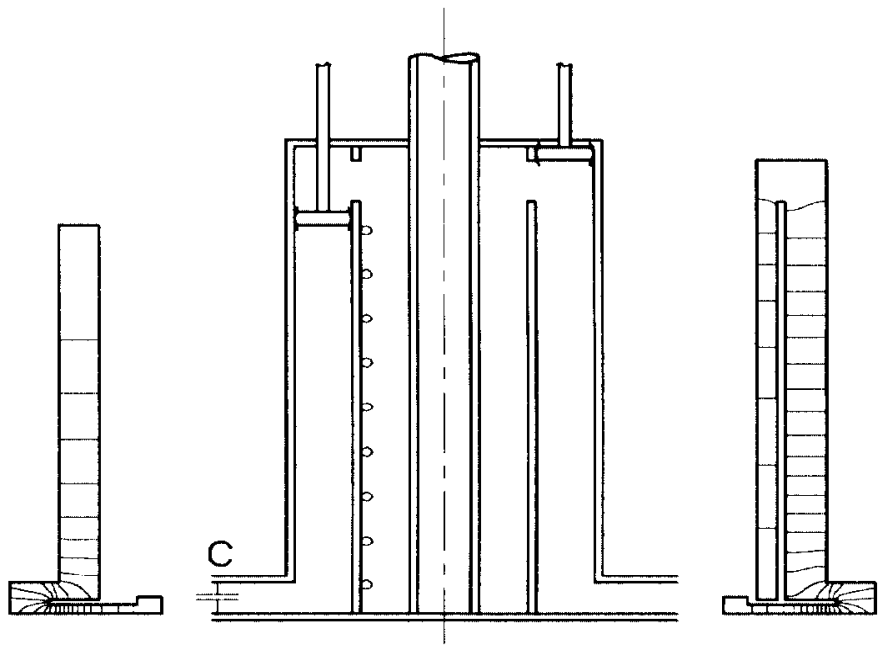

Fig. 2. High/low frequency modes. 


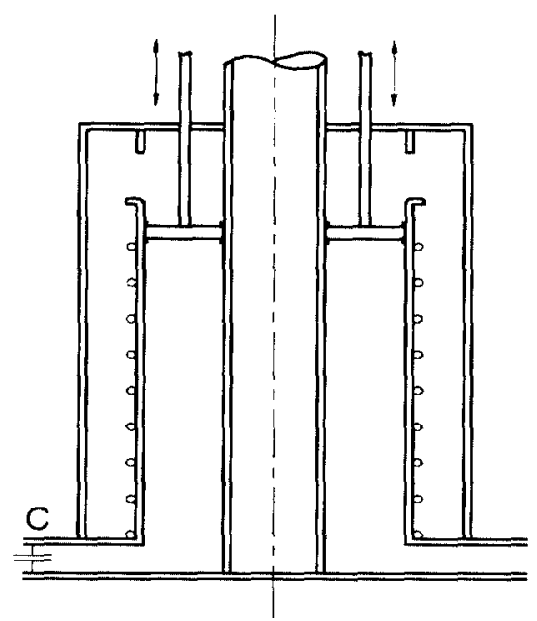

Fig. 3. Alternative concept.

The choice of the best concept for given boundary conditions depends mainly on the space available for the resonator system, the amplitude of $\mathrm{rf}$ voltage at both frequencies, and which mode of operation will be prevailing in production runs. The designer will then optimize the cavity dimensions within the available space. The power loss in the system is strongly affected by the characteristic impedance of the coaxial sections which in turn depends on the radial dimensions. A comparison below illustrates the effects of individual parameters for the two configurations, and it could serve as a guide when selecting a real system.

\section{BASIC THEORY}

For a shorted transmission line of characteristic impedance $Z$ and length $l$, which is used to resonate the equivalent dee capacity $\mathrm{C}$ (capacitively loaded $\lambda / 4$ resonator) the following equations apply (high frequency approximation, dielectric losses $\rightarrow 0$ ):

$$
C=\frac{1}{\omega Z \operatorname{tg} \beta l}
$$

$Z=60 \ln (D / d)$, and $\beta$ is the phase factor.

When two transmission lines are used in series with characteristic impedances $Z_{1}$ and $Z_{2}$ of corresponding lengths $l_{1}$ and $l_{2}$ (Fig. 4), resonance occurs when

$$
\frac{1}{\omega C}=\frac{Z_{1} \operatorname{tg} \beta l_{1}+Z_{2} \operatorname{tg} \beta l_{2}}{1-\frac{Z_{1}}{Z_{2}} \operatorname{tg} \beta l_{1} \operatorname{tg} \beta l_{2}}
$$

For the folded transmission line $l 1 \sim l 2$, and neglecting the wall thickness of the intermediate conductor we have

$$
\frac{1}{\omega C}=\frac{Z_{1}+Z_{2}}{1-\frac{Z_{1}}{Z_{2}} \operatorname{tg} \beta l}
$$

Expressing the length $/$ from Eq. 3.

$$
l=\frac{1}{\beta} \operatorname{arctg}\left(\frac{Z_{1}+Z_{2}}{Z_{2}-Z_{1}} \omega C Z_{2}\right)
$$

The rf power dissipated in the folded transmission line is

$$
P=\frac{R_{1} I_{0}^{2}}{8 \beta}(2 \beta l+\sin 2 \beta l)_{0}^{l}+\frac{R_{2} I_{3}^{2}}{8 \beta}(2 \beta l+\sin 2 \beta l)_{l}^{2 l} \uparrow
$$

where $R_{1}$ and $R_{2}$ are the distributed line resistances.

The above equations were tested by measurements on physical models and by SUPERFISH. There the accuracy is mostly affected by modelling the simplified dee which for the code has to be represented in axial symmetry as opposed to a true 3-D case.

\section{MODEL MEASUREMENTS}

The measurements were taken on a simple 1:1 wooden, copper-clad model, as in Fig 2.

With conductor radii of $10 / 5.1 / 3 \mathrm{~cm}$ the line impedances were $Z_{s}=40 \Omega, Z_{1}=32 \Omega$. Measured values are in Table 1.

Table 1. Data measured on model.

\begin{tabular}{lcc}
\hline \hline Measured: & $\mathrm{A}$ & $\mathrm{B}$ \\
\hline Frequency $\overline{\mathrm{f}}[\overline{\mathrm{M}} \mathrm{Hz}]$ & 73.7 & 36.8 \\
Stem length $\mathrm{l}_{r}[\mathrm{~cm}]$ & 39.0 & $2 \times 70.5$ \\
Voltage $\mathrm{V}_{c}[\mathrm{kV}]$ & 23.2 & 38.0 \\
\hline \hline
\end{tabular}

All quantities are normalized to dee voltage equal to $50 \mathrm{kV}$ at 74 $\mathrm{MHz}$ and $25 \mathrm{kV}$ at $37 \mathrm{MHz}$.

From the above results the relevant system parameters are computed at both frequencies, and the results summarized in Table 2 .

Table 2. Results computed from measurements.

\begin{tabular}{llcc}
\hline & $\begin{array}{c}\text { Current at short } \\
{[\mathrm{A}]}\end{array}$ & $\begin{array}{c}\text { Power } \\
{[\mathrm{kW}]}\end{array}$ \\
\hline $74 \mathrm{MHz}$ & analytically & 1564 & 4.60 \\
& SUPERFISH & 1480 & 4.30 \\
$37 \mathrm{MHz}$ & analytically & 747 & 3.40 \\
& SUPERFISH & 754 & 3.45 \\
\hline \hline
\end{tabular}

\$Shorting plate not included.

The analytic treatment of the problem yields sufficiently accurate results for the design of a folded stem accelerating system.

\section{COMPARISON OF CONCEPTS}

Assume a dual frcqucncy acceleration system with a limit of $200 \mathrm{~mm}$ on the outer conductor (I.D.), dee capacity $74 \mathrm{pF}$, operating at 36.8 and $73.7 \mathrm{MHz}$.

Note: The values obtained from Eq. 5 represent one quarter of the power for the whole accelerating system. They do not include the power loss in the dee region and in the shorting planes, nor do they include any losses due to imperfect contacts between if parts. The real rf loss is $\approx 20 \%$ higher than calculated. The $r f$ power delivered to the beam must also be added as ohmic load. 


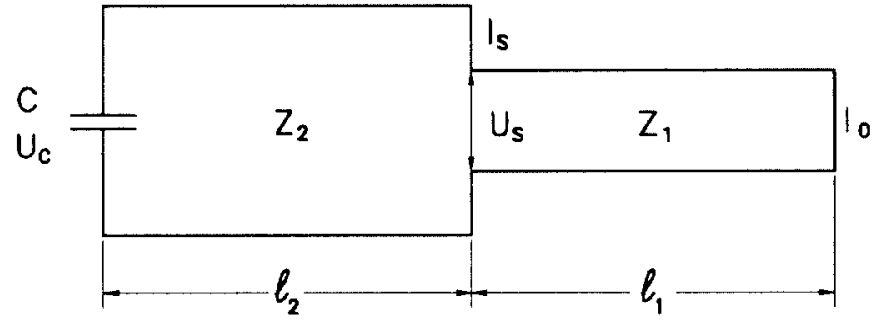

Fig. 4. Folded line equivalent circuit.

At the low frequency the two concepts under comparison are in Figs. 2 and 3. The equivalent circuits are the same as in Fig. 4.

The two configurations are schematically shown in the corresponding sketches, and the results obtained analytically for both operating frequencies are summarized in Table 3 .

Table 3. Comparison of concepts (Figs. 2 and 3).

\begin{tabular}{lcc}
\hline Parameter & \multicolumn{2}{c}{ Configuration } \\
\cline { 2 - 3 } & Fig. 2. & Fig. 3. \\
\hline
\end{tabular}

Frequency $\mathrm{f}=73.7 \mathrm{MHz}$; dee voltage $=50 \mathrm{kV}$

$\begin{array}{lcc}\text { Line impedance } \mathrm{Z}_{\circ}[\Omega] & 55 & 40 \\ \text { Current amplitude } \mathrm{I}_{\circ}[\mathrm{A}] & 1278 & 1616 \\ \text { Power loss calc. Pc }[\mathrm{kW}] & 3.9 & 3.7 \\ \text { Expected Pe }[\mathrm{kW}] & 5 & 4.5\end{array}$

Frequency $\mathrm{f}=36.8 \mathrm{MHz}$; dee voltage $=25 \mathrm{kV}$

Line impedances $[\Omega] \quad \mathrm{Z}_{1}=17.3 \quad \mathrm{Z}_{0}=55 \quad \mathrm{Z}_{1}=30 \quad \mathrm{Z}_{0}=40$

Current amplitudes [A] $\quad \mathrm{I}_{s}=637 \mathrm{I}_{0}=778 \quad \mathrm{I}_{s}=640 \mathrm{I}_{0}=747$

Power loss calc. $[\mathrm{kW}] \quad \mathrm{P}_{\mathrm{c}=2.1} \quad \mathrm{P}_{\mathrm{c}=3.3}$

Expected $[k W] \quad P e=3.0 \quad P e=4.0$

Note: For the complete system the power loss is multiplied by four.

The results show that both configurations represent $v i-$ able design options. The difference in the capital cost of both variants will be of little importance, and the param- eters most affecting the choice would be the outer dimensions, and power consumption. In comparison to a system with straight stems (Ref. [1]) in both configurations the overall length is $\approx 25 \%$ less, and the respective power losses are about equal at the high frequency, and 20 or $50 \%$ higher in the low frequency mode.

\section{RESONATORS WITH MULTIPLE FOLDS}

It is obvious that the idea of using nested cylindrical conductors can be extended to include multiple folds. For the same diameter of the outermost conductor the resonator is then further shortened at the expense of higher power losses. An example of such resonator with two moveable shorts is in Fig. 5, again showing the rf field distribution obtained by SUPERFISII. Practical applications of these resonators are promising, and they will be explored in the future.

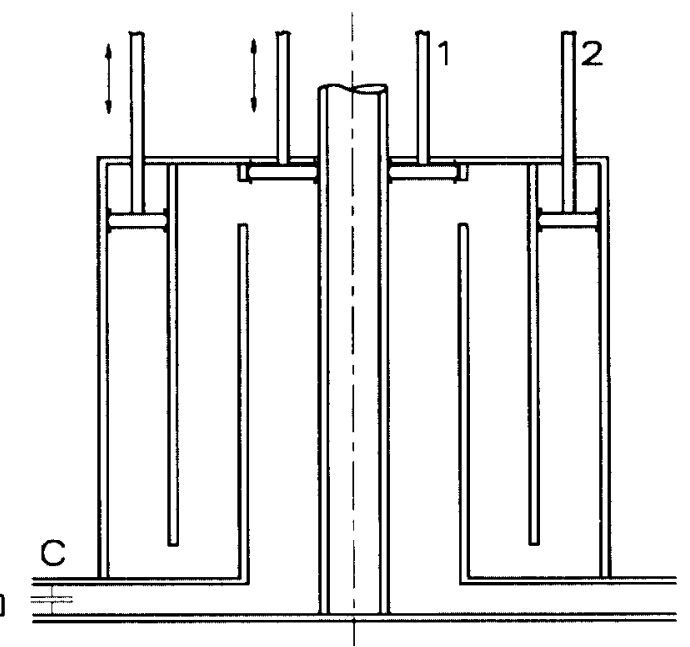

Fig. 5. Line with two folds.

\section{REFERENCE}

[1] M. Eiche, K.L. Erdman, P. Lanz, M. Lipnicki, and M. Zach, Dual frequency resonator system for a compact cyclotron, Proc. XIII Intern. Conf. on Cyclotrons and Their Applications, (World Scientific, Singapore, 1992) p. 515 . 